\title{
Anesthesia Comlications After Neurosurgical Procedure in Post-Anesthesia Care Unit Running Title: Complications in Post-Anesthesia Care Unit
}

\author{
Mingkai Chen \\ West China Hospital of Sichuan University \\ Juan Liu \\ West China Hospital of Sichuan University \\ Xuechao Hao \\ West China Hospital of Sichuan University \\ Mengchan $\mathrm{Ou}$ \\ West China Hospital of Sichuan University \\ Xindi Song \\ West China Hospital of Sichuan University \\ Yu Li (D liyu1936@163.com ) \\ West China Hospital of Sichuan University
}

\section{Research Article}

Keywords: anesthesia, hypertension, hypoxia, neuralgia, aneurysm

Posted Date: March 25th, 2021

DOI: https://doi.org/10.21203/rs.3.rs-334278/v1

License: (c) (i) This work is licensed under a Creative Commons Attribution 4.0 International License. Read Full License 


\section{Abstract}

Background: Complications in anesthesia emergence after neurosurgery varies widely but is associated with negative neurological outcomes. The present study was to investigate postoperative complication in the PACU.

Methods: During July 2015 to July 2016, the retrospective cohort study was conducted and 1586 adult patients who underwent selective neurological surgery in West China Hospital, Sichuan University, were recruited. The complications, including hypertension, hypoxia, etc. were determined by an anesthetist in PACU. Univariate statistics were performed using the chi-squared test, and $95 \%$ confidence intervals were determined for the resultant risk ratios. A multivariate model was constructed using significant variables from the univariate analysis $(\mathrm{p}<0.05)$ with binary logistic regression.

Results: Among the involved 1586 patients, the most frequent complications were hypertension ( $21.5 \%$ of patients) and hypoxemia (7.25\% of patients), followed by airway obstruction (6.87\%). Top three surgery for hypertension incidence were surgery for trigeminal neuralgia, aneurysm, subtentorial lesions. Significant predictors of hypertension included advanced age, sex, preoperative hypertension, and duration of general anesthesia.

Conclusions: In the period of anesthesia recovery of neurosurgical patients, hypertension, hypoxemia, airway obstruction are three main complications in PACU.

\section{Introduction}

Postoperative complication during anesthesia emergence greatly increase morbidity and mortality as well as the financial burden on health care, mainly by prolonging the hospital stay and the rate of readmission and/or reoperation[1]. Patients who experienced postoperative complications are more likely to experience hospital-acquired conditions, thus being more than twice as likely to die in the hospital as those who did not experience one. A single complication increased the mean total cost by $\$ 10,000-\$ 20,000$, and with 3 complications this cost increased by 3 -fold[2-6]. Besides the well-studied and preventable common complications such as surgical site infections and urinary tract infection, complications of certain types of surgeries that may require more specialized management should also be paid more attention-for example, blood pressure needs to be controlled within a specified range in aneurysm patients. In the neurosurgery setting it is reported that patients, especially after craniotomy, have a relatively higher incidence of complications[7-11], including neurological adverse events (new motor deficit, dysphasia, seizure, deterioration of consciousness), hemodynamic adverse events (bradycardia, hypertension, hypotension), respiratory adverse events, postoperative nausea and vomiting, metabolic adverse events, hemorrhage, hyperthermia, pain, and reoperation[11]. In the past, patients undergoing neurosurgery were often directly admitted to an intensive care unit, but studies show increasingly that patients with better perioperative conditions can be monitored safely in the postanesthesia care unit (PACU)[12-14]. The PACU provides general to intensive care to immediate 
postsurgical patients until their condition has stabilized and they can be transferred to the general ward. It is well recognized that PACU-oriented care can effectively prevent early complications and decrease the average hospital stay, thus freeing up the availability of the operating room.

To reduce complications, we need to identify predictive risk factors of possible occurrence. Therefore, in this study we investigate the incidence of complications in the PACU after neurological surgery among 1586 patients to help us improve patients' well-being.

\section{Methods}

This single center retrospective cohort study was proved by Ethical Committee of West China Hospital, Sichuan University (Ref:2017173) on July 6, 2017 with a waiver of informed consent because of the nature of the retrospective cohort study. Patients, aged $>18 \mathrm{y}$, undergoing selective neurosurgery during the period July 2015 through July 2016 was recruited. Non-elective patients went to the ICU after surgery were excluded from this study. Data collected included general demographics, patients' preoperative comorbidities, type of surgery, duration of anesthesia, and postoperative complications that occurred in the PACU. Perioperative management was standardized according to institutional guidelines and regulations. Regular fasting and water-deprivation was applied to each patient. For patients who have the history of hypertension, anti-hypertensive medication was given until the operation day. Reserpine was replaced by regular antihypertensive drugs one week in advance. General anesthesia was administered with intravenous midazolam $(0.05 \mathrm{mg} / \mathrm{kg})$, cisatracurium $(0.15-0.2 \mathrm{mg} / \mathrm{kg}$ ), propofol (2 $\mathrm{mg} / \mathrm{kg})$, and sufentanil ( $0.3 \mu \mathrm{g} / \mathrm{kg}$ initial bolus, repeated $0.1-0.2 \mu \mathrm{g} / \mathrm{kg}$ hourly). Target-controlled infusion of propofol $(3-4 \mu \mathrm{g} / \mathrm{mL})$ and remifentanil $(0.1-0.2 \mu \mathrm{g} / \mathrm{kg} / \mathrm{min})$ were used for maintenance of anesthesia and adjusted to maintain the mean arterial pressure (MAP) within $60-80 \mathrm{mmHg}$. Ventilation was mechanically controlled to achieve an end-tidal $\mathrm{CO} 2$ of $30-35 \mathrm{mmHg}$. Other intraoperative monitoring included electrocardiogram, heart rate, arterial blood pressure, oxygen saturation, arterial blood gases, and urine output. After surgery, patients were transferred to the PACU under endotracheal intubation. Upon recovery of spontaneous breathing, $1 \mathrm{mg}$ of neostigmine and $0.5 \mathrm{mg}$ of atropine were administered to eliminate the effect of muscle relaxants, unless contraindicated. All patients were monitored for at least $1 \mathrm{~h}$ in the PACU and then transferred to the ward.

Pain management: The incision was infiltrated with ropivacaine before sutured. Sufentanil was used as rescue analgesic in PACU. If the measured visual analogue scale (VAS) was $>4$ or patients requested analgesia, the rescue analgesia was administered as an intravenous injection of sufentanil $5 \mu \mathrm{g}$, repeatedly at a minimal interval of $10 \mathrm{~min}$ if needed until VAS was $<4$. The complications happened in PACU were defined as following: Hypotension and hypertension: either systolic blood pressure (SBP) or diastolic blood pressure (DBP), decreases or increases of $20 \%$ or more over the baseline, respectively.

Arrhythmia: an irregular heartbeat, including tachycardia (higher than 120 beats per minute), bradycardia (slower than 60 beats per minute) or irregular heartbeat (flutter, fibrillation or premature contraction). Hypoxemia: oxygen saturation (SpO2) values under 90\% while breathing air. Upper airway obstruction: 
mainly caused by loss of muscle tone present in the awake state, including laryngeal spasm and glossoptosis. [15] Pain: A 11-point pain scale was used to assess pain, where $0-3$ is mild pain, 4-7 is moderate pain and 8-10 is severe pain.[16]Emergence agitation: including emergence delirium or emergence excitement PONV (Postoperative Nausea and vomiting): emesis and queasiness occurring after general anesthesia Postoperative shivering: involuntary contraction or twitching of the muscles after surgery due to low body temperature. Seizures: clinical manifestations include abnormal motor, sensory and psychic phenomena.

\section{Statistical Analysis}

Normally distributed continuous variables were present as means \pm stand deviation, and the student' $t$ test and chi-squared test were used for statistical analysis. Non-normally distributed continuous variables were presented as median (quartile range) and were analyzed with Mann-Whitney $\mathrm{U}$ test or KruskalWallis test. Multiple logistic regression analysis was performed to determine the variables associated with hypertension.

\section{Results}

A total of 1586 adult patients scheduled for neurosurgery under general anesthesia were recruited (July 2015-July 2016). Mean patient age was $50 \pm 13$ years, body weight $61 \pm 11 \mathrm{~kg}$, and sex ratio (F/M) 735:851. The duration of surgery, anesthesia, and PACU stay was $140[95,200], 210[155,270]$, and 85[65, 115] min, respectively. Demographics are outlined in Table 1. The major anesthesia complication for neurosurgery in the PACU is hypertension, which occurred in $21.5 \%$ of neurosurgical patients (Table 2). The second and third most common complications were hypoxemia and airway obstruction, accounting for $7.25 \%$ and $6.87 \%$, respectively. We found no difference in demographic characteristics between hypertensive and non-hypertensive patients in PACU(Table 3). Among the 250 patients who had hypertension before surgery, 144 were hypertensive in the PACU, accounting for $57.6 \%$. Significant independent risk factors for hypertension consisted of history of hypertension (odds ratio [OR] 6.29, 95\% confidence interval [Cl] 4.58-8.64), sex (OR 1.62, 95\% Cl 1.23-2.12), advanced age (OR $1.45,95 \% \mathrm{Cl}$ 1.29-1.62), and duration of general anesthesia (OR 1.33, 95\% $\mathrm{Cl} 1.12-1.57$ )(Table 4). The incidence of postoperative hypertension in the PACU according to surgical procedure also are showed in Table 5. 
Table 1

Demographic characteristics of 1586 adult patients undergoing neurosurgery

\section{Characteristics}

Sex ration (M/F)
Age (year)
Weight $(\mathrm{kg})$
ASA classification

।

$\|$

III

IV

$\mathrm{V}$

Surgery $(\min )$

Anesthesia ( $\mathrm{min})$

PACU (min)

Spinal

Cranial

Aneurysm

Supratentorial lesions

Subtentorial lesions

Sellar region lesions

Trigeminal neuralgia

Hemifacial spasm

Pakinson disease

Hydrocephalus

Epilepsy

Others
Adult

$(n=1586)$

$735 / 851$

$50 \pm 13$

$61 \pm 11$

8

838

707

28

5

$140[95,200]$

$210[155,270]$

$85[65,115]$

336

1250

182

434

162

155

24

68

48

24

21

132

Data are listed as"value (\%)"and or median [25-75 percentiles]. COPD chronic obstructive pulmoriary disease; M/F Male/Female 


\begin{tabular}{|ll|}
\hline Characteristics & $\begin{array}{l}\text { Adult } \\
(\mathbf{n}=\mathbf{1 5 8 6})\end{array}$ \\
\hline Comorbidities & 250 \\
\hline Hypertension & 40 \\
\hline Diabetes & 40 \\
\hline Coronary disease (myocardial infraction) & 36 \\
\hline Neurological disease & 33 \\
\hline Respiratory disease (COPD) & 16 \\
\hline Difficult airway & 1 \\
\hline Cirrhosis & \\
\hline $\begin{array}{l}\text { Data are listed as“value (\%)"and or median [25-75 percentiles]. COPD chronic obstructive pulmoriary } \\
\text { disease; M/F Male/Female }\end{array}$ \\
\hline
\end{tabular}

Table 2

Incidence of complication in 1586 neurosurgical cases in the PACU

\begin{tabular}{|ll|}
\hline Comlication & No. (\%) of incidences \\
\hline Cardiovascular & \\
\hline Hypertension & $341(21.5)$ \\
\hline Hypotension & $14(0.88)$ \\
\hline Arrhythmia & $43(2.71)$ \\
\hline Respiratorty & $115(7.25)$ \\
\hline Hypoemia & $109(6.87)$ \\
\hline Airway obstruction & $5(0.32)$ \\
\hline Others & $51(2.33)$ \\
\hline Pain & $37(2.33)$ \\
\hline Emergence agitation & $28(1.77)$ \\
\hline Nausea/vomiting & $18(1.13)$ \\
\hline Postoperative shivering & $2(0.13)$ \\
\hline Seizures & \\
\hline Data are listed as “value (\%)". \\
\hline
\end{tabular}


Table 3

Demographic characteristics of hypertensive and non-hypertensive patients

\begin{tabular}{|lll|}
\hline Variables & $\begin{array}{l}\text { Hypertension } \\
(\mathbf{n}=341)\end{array}$ & Non-hypertension $(\mathbf{n}=\mathbf{1 2 4 5})$ \\
\hline Sex ratio (M/F) & $128 / 213$ & $607 / 638$ \\
\hline Age (year) & $56.4 \pm 11.1$ & $48.2 \pm 13.6$ \\
\hline Weight (kg) & $61.3 \pm 10.7$ & $61.3 \pm 10.8$ \\
\hline Hypertensive Comorbidities & $144 / 341$ & $106 / 1245$ \\
\hline ASA & & \\
\hline I & 2 & 6 \\
\hline II & 144 & 694 \\
\hline III & 185 & 522 \\
\hline IV & 10 & 18 \\
\hline V & 0 & 5 \\
\hline Surgery (min)* & $151[100,208]$ & $145[100,200]$ \\
\hline Anesthesia (min) & $215[162,280]$ & $210[162,270]$ \\
\hline PACU (min) & $100[75,132]$ & $85[65,110]$ \\
\hline Data are listed as means \pm stand deviation and or median [25-75 percentiles]. \\
\hline
\end{tabular}

Table 4

Risk factors for hypertension occurring in the PACU

\begin{tabular}{|llll|}
\hline Variables & OR & $95 \% \mathrm{Cl}$ & p-value $^{*}$ \\
\hline Sex & 1.62 & $1.23-2.12$ & 0.001 \\
\hline Age & 1.45 & $1.29-1.62$ & $<0.001$ \\
\hline Hypertension history & 6,29 & $4.58-8.64$ & $<0.001$ \\
\hline The duration of general anesthesia & 1.33 & $1.12-1.57$ & 0.001 \\
\hline ASA & 0.97 & $0.75-1.25$ & 0.805 \\
\hline Surgical site & 1.035 & $0.96-1.11$ & 0.357 \\
\hline *Multivariate analysis & & & \\
\hline
\end{tabular}


Table 5

Incidence of postoperative hypertension in the PACU according to surgical procedure

\begin{tabular}{|lll|}
\hline Indication for surgery & Hypertension, $\mathrm{n}(\%)$ & Non-hypertension, $\mathrm{n}(\%)$ \\
\hline Spinal & $54(16.1)$ & $282(83.9)$ \\
\hline Cranial & $287(23)$ & $963(77)$ \\
\hline Trigeminal neuralgai & $14(58.3)$ & $10(42.7)$ \\
\hline Aneurysm & $79(43.4)$ & $103(56.6)$ \\
\hline Subtentorial lesions & $51(31.5)$ & $111(68.5)$ \\
\hline Sellar region lesions & $38(24.5)$ & $117(75.5)$ \\
\hline Parkinson disease & $11(22.9)$ & $37(87.1)$ \\
\hline Hydrocephalus & $4(16.7)$ & $20(83.3)$ \\
\hline Hemifacial spasm & $11(16.2)$ & $57(85.3)$ \\
\hline Supratentorial lesions & $64(14.7)$ & $370(85.3)$ \\
\hline Epilepsy & $1(4.8)$ & $20(95.2)$ \\
\hline Others & $14(10.6)$ & $118(89.4)$ \\
\hline Data are listed as“value (\%)" & \\
\hline
\end{tabular}

\section{Discussion}

Regarding neurosurgical characteristics, stable awake status and recovery of neural function are required as soon as possible to facilitate early neurological examination and improve the neurological outcome. Studies show that overall complications after neurosurgery are relatively high[7-11]. In our research we analyzed data of 1586 adult patients over a period of 1 year to obtain a better understanding of postoperative complications in neurosurgery.

The main finding of our study is that the most frequent complication was hypertension, followed by hypoxemia and airway obstruction. The thresholds used in clinical hypertension have included either SBP $>160 \mathrm{mmHg}, \mathrm{DBP}>90 \mathrm{mmHg}, \mathrm{MAP}>110 \mathrm{mmHg}$, or a relative change from baseline (an increase in SBP or DBP of $\geq 20 \%$ ). In specific cases such as ruptured aneurysm, patients may already have hypertension before surgery. For this reason we used the increase in SBP or DBP of $\geq 20 \%$ as the threshold to treat hypertension.

For neurological patients, hypertension in the perioperative and postoperative period may increase intracranial pressure and cause bleeding at the surgical site, intracranial hemorrhage, or cerebral ischemia, resulting in poor outcomes that include prolonged hospital stay, brain dysfunction, and 
increased mortality, especially in the first 24 hours[17-20]. One study found that post-craniotomy intracranial hematoma was associated with intraoperative and postoperative hypertension[21]. In our study the incidence of hypertension was $23 \%$ in cranial cases and $16 \%$ in spinal cases, similar to the findings from a study by Rolston et al. in which cranial cases were more likely to have complications than spinal cases[9]. This may be explained by the nature of disease requiring cranial neurosurgery being very different from that requiring spine surgery, whereby the former is often much more liable to operative complications. In addition, acute physiological changes during anesthesia recovery (such as increased cerebral blood flow and intracranial pressure) can add to the severity of intracranial complications[7].

In our study, we found trigeminal neuralgia is the most common surgery type leading to hypertension. It may due to that the patients who suffer from trigeminal neuralgia have a high frequent of a concomitant hypertension, which is called neurogenic essential hypertension[22, 23]. Besides, the severe pain of this disease may stimulate the sympathetic system, thus increasing the blood pressure.

Not surprisingly, aneurysm patients are second most frequently affected by hypertension. For patients with aneurysmal subarachnoid hemorrhage, preceding hypertension is an independent risk factor for poor outcome and cerebral infarction[24]. Research has also indicated that normalization of blood pressure will significantly reduce the incidence of aneurysm rupture, while partial normalization of blood pressure will lead to a trend of reduced rupture rate. Overall, therefore, there appears to be a dose-dependent relationship between blood pressure reduction and prevention of aneurysmal rupture[25].

It should be noted that some factors such as pain, anxiety, ureter discomfort, hypothermia, and hypoxia are associated with postoperative hypertension and should be excluded before administering antihypertensive drugs. In our study, postoperative moderate to severe pain occurred in 51 of the patients $(3.22 \%)$ in the PACU, almost half of whom (45.1\%) had hypertension. Research shows that the MAP is higher in patients with severe postoperative pain than in those with no or mild pain[26]. In proportion to the severity of the stimulus, the spinal reflexes recruited by increasing pain may cause activation of the sympathetic nervous system, which increases peripheral resistance[27].

The general incidence of postoperative nausea and vomiting (PONV) is about $30-50 \%$, and up to $80 \%$ in high-risk groups without antiemetic prophylaxis after surgery[28-31]. Owing to changes in intracranial pressure, cerebral intravascular pressure, hemostasis, and cerebral perfusion, the incidence of PONV in craniotomy surgery is higher[32]. Since it is a frequently occurring postoperative complication in the neurosurgery patient population, a variety of antiemetic agents is used to lower the incidence of PONV, such as 5-HT3 receptor antagonists, dexamethasone, metoclopramide, and the neurokinin-1 receptor blocker aprepitant[33, 34]. In our study, PONV problems occurred in $1.77 \%$ of patients, For which we use tropisetron, a serotonin antagonist used in the prevention of chemotherapy-induced nausea and vomiting.

In our study, the risk factors of hypertension such as hypertensive comorbidities, sex, advanced age, and the duration of general anesthesia are traditional predictors. Since research on the risk factors for postoperative hypertension remain inadequate, further investigation is warranted. 
This study has some limitations. First and most importantly, it is an observational study and therefore can only address association but not causality. Second, because most emergence operations went to ICU after surgery, so we only included elective neurosurgery, while most hypertensive cerebral hemorrhage patients, who make up a sizable proportion of the general hypertensive population, undergo surgery in the emergency operating room.

\section{Conclusions}

In the period of anesthesia recovery of neurosurgical patients, hypertension, hypoxemia, airway obstruction are three main complications in PACU.

\section{Abbreviations}

PACU: Post-anesthesia care unit

ICU: Intensive care unit

MAP: Mean arterial pressure

SBP: Systolic blood pressure

DBP: Diastolic blood pressure

C02: Carbon dioxide

VAS: Visual analogue scale

$\mathrm{SpO}_{2}$ : Oxygen saturation

PONV: Postoperative nausea and vomiting

OR: Odd ration

5-HT: 5-hydroxytryptamine

Cl: Confidence interval

\section{Declarations}

\section{Availability of data and materials}

The datasets during and/or analysed during the current study available from the corresponding author on reasonable request. 
All the clinicians who helped in data collection.

\section{Funding}

The authors received no funding for this work.

Competing Interests

The authors declare no conflict of interests.

Author information

Affiliations

Department of Anesthesiology, West China Hospital of Sichuan University, Chengdu, Sichuan, China Mingkai Chen, Juan Liu, Xuechao Hao \& Yu Li

Laboratory of Anesthesia \& Critical Care Medicine, Translational Neuroscience Center, and Department of Anesthesiology, West China Hospital of Sichuan University, Chengdu, Sichuan, China

Mengchan Ou

West China School of Medicine, Sichuan University, Chengdu, Sichuan, China

Xindi Song

Corresponding author

Correspondence to Yu Li.

Ethics declarations and consent to participate

This observational study was approved by Ethical Committee of West China Hospital, Sichuan University (Ref:2017173) on July 6, 2017, and adhered to the Strengthening the Reporting of Observational studies in Epidemiology (STROBE) guidelines. Because of retrospective study, informed consent was waived.

Consent for publication

Not Applicable.

\section{References}

1. Sharma PT, Sieber FE, Zakriya KJ et al: Recovery room delirium predicts postoperative delirium after hip-fracture repair. Anesth Analg 2005, 101(4). 
2. Vonlanthen R, Slankamenac K, Breitenstein $S$ et al: The impact of complications on costs of major surgical procedures: a cost analysis of 1200 patients. Ann Surg 2011, 254(6):907-913.

3. Patel AS, Bergman A, Moore BW et al: The economic burden of complications occurring in major surgical procedures: a systematic review. Appl Health Econ Health Policy 2013, 11(6):577-592.

4. Sharma M, Sonig A, Ambekar S et al: Discharge dispositions, complications, and costs of hospitalization in spinal cord tumor surgery: analysis of data from the United States Nationwide Inpatient Sample, 2003-2010. J Neurosurg Spine 2014, 20(2):125-141.

5. Zacharia BE, Deibert C, Gupta G et al: Incidence, cost, and mortality associated with hospital-acquired conditions after resection of cranial neoplasms. Neurosurgery 2014, 74(6):638-647.

6. Nandyala SV, Elboghdady IM, Marquez-Lara A et al: Cost analysis of incidental durotomy in spine surgery. Spine (Phila Pa 1976) 2014, 39(17):E1042-E1051.

7. Bruder NJ: Awakening management after neurosurgery for intracranial tumours. Curr Opin Anaesthesiol 2002, 15(5):477-482.

8. Marini $H$, Merle V, Derrey $S$ et al: Surveillance of unplanned return to the operating theatre in neurosurgery combined with a mortality-morbidity conference: results of a pilot survey. BMJ Qual Saf 2012, 21(5):432-438.

9. Rolston JD, Han SJ, Lau CY et al: Frequency and predictors of complications in neurological surgery: national trends from 2006 to 2011. J Neurosurg 2014, 120(3):736-745.

10. Cote DJ, Karhade AV, Larsen AMG et al: United States neurosurgery annual case type and complication trends between 2006 and 2013: An American College of Surgeons National Surgical Quality Improvement Program analysis. J Clin Neurosci 2016, 31:106-111.

11. Lonjaret L, Guyonnet M, Berard E et al: Postoperative complications after craniotomy for brain tumor surgery. Anaesth Crit Care Pain Med 2017, 36(4):213-218.

12. Kelly DF: Neurosurgical postoperative care. Neurosurg Clin N Am 1994, 5(4):789-810.

13. Beauregard CL, Friedman WA: Routine use of postoperative ICU care for elective craniotomy: a costbenefit analysis. Surg Neurol 2003, 60(6).

14. Rhondali $O$, Genty $C$, Halle $C$ et al: Do patients still require admission to an intensive care unit after elective craniotomy for brain surgery? J Neurosurg Anesthesiol 2011, 23(2):118-123.

15. Hillman DR, Platt PR, Eastwood PR: The upper airway during anaesthesia. Br J Anaesth 2003, 91(1):31-39.

16. Ramsay MA: Acute postoperative pain management. Proc (Bayl Univ Med Cent) 2000, 13(3):244247.

17. Broderick J, Connolly S, Feldmann E et al: Guidelines for the management of spontaneous intracerebral hemorrhage in adults: 2007 update: a guideline from the American Heart Association/American Stroke Association Stroke Council, High Blood Pressure Research Council, and the Quality of Care and Outcomes in Research Interdisciplinary Working Group. Stroke 2007, 38(6):2001-2023. 
18. Elliott J, Smith M: The acute management of intracerebral hemorrhage: a clinical review. Anesth Analg 2010, 110(5):1419-1427.

19. Shanbhag NC, Henning RH, Schilling L: Long-term survival in permanent middle cerebral artery occlusion: a model of malignant stroke in rats. Sci Rep 2016, 6:28401.

20. Zhou H, Sun J, Ji X et al: Correlation Between the Integrity of the Circle of Willis and the Severity of Initial Noncardiac Cerebral Infarction and Clinical Prognosis. Medicine (Baltimore) 2016, 95(10):e2892.

21. Jian M, Li X, Wang A et al: Flurbiprofen and hypertension but not hydroxyethyl starch are associated with post-craniotomy intracranial haematoma requiring surgery. $\mathrm{Br} J$ Anaesth 2014, 113(5):832-839.

22. Teruel A, Ram S, Kumar SKS et al: Prevalence of hypertension in patients with trigeminal neuralgia. J Headache Pain 2009, 10(3):199-201.

23. Pan SL, Yen MF, Chiu YH et al: Increased risk of trigeminal neuralgia after hypertension: a populationbased study. Neurology 2011, 77(17):1605-1610.

24. Juvela S, Siironen J, Kuhmonen J: Hyperglycemia, excess weight, and history of hypertension as risk factors for poor outcome and cerebral infarction after aneurysmal subarachnoid hemorrhage. $J$ Neurosurg 2005, 102(6).

25. Tada $\mathrm{Y}$, Wada $\mathrm{K}$, Shimada $\mathrm{K}$ et al: Roles of hypertension in the rupture of intracranial aneurysms. Stroke 2014, 45(2):579-586.

26. Ledowski T, Reimer M, Chavez $\mathrm{V}$ et al: Effects of acute postoperative pain on catecholamine plasma levels, hemodynamic parameters, and cardiac autonomic control. Pain 2012, 153(4):759-764.

27. Saccò M, Meschi M, Regolisti $G$ et al: The relationship between blood pressure and pain. J Clin Hypertens (Greenwich) 2013, 15(8):600-605.

28. Wiesmann T, Kranke P, Eberhart L: Postoperative nausea and vomiting - a narrative review of pathophysiology, pharmacotherapy and clinical management strategies. Expert Opin Pharmacother 2015, 16(7):1069-1077.

29. Hellickson JD, Worden WR, Ryan C et al: Predictors of Postoperative Nausea and Vomiting in Neurosurgical Patients. J Neurosci Nurs 2016, 48(6):352-357.

30. Cao X, White PF, Ma H: An update on the management of postoperative nausea and vomiting. J Anesth 2017, 31(4):617-626.

31. Tsaousi GG, Pourzitaki C, Bilotta F: Prophylaxis of postoperative complications after craniotomy. Curr Opin Anaesthesiol 2017, 30(5):534-539.

32. Tsutsumi YM, Kakuta N, Soga T et al: The effects of intravenous fosaprepitant and ondansetron for the prevention of postoperative nausea and vomiting in neurosurgery patients: a prospective, randomized, double-blinded study. Biomed Res Int 2014, 2014:307025.

33. Kovac AL: Update on the management of postoperative nausea and vomiting. Drugs 2013 , 73(14):1525-1547. 
34. Singh PM, Borle A, Rewari V et al: Aprepitant for postoperative nausea and vomiting: a systematic review and meta-analysis. Postgrad Med J 2016, 92(1084):87-98. 